\title{
ANALYSIS OF COUPLED EFFECT OF PERTINENT PROCESS PARAMETERS OF WATER ELECTROLYSIS
}

\author{
B. Mandal ${ }^{1}$, A. Sirkar ${ }^{1}$, Parameswar De ${ }^{2}$, S. J. Sahu ${ }^{1}$, A. Ghosh ${ }^{1}$ \\ ${ }^{1}$ Department of Chemical Engineering, Haldia Institute Of Technology, Haldia - 721 657, West Bengal, India. \\ 2 Department of Chemical Engineering, University Of Calcutta, 92 A.P.C. Road, Kolkata - 700 009, India.
}

\begin{abstract}
Water electrolysis for the production of hydrogen proceeds via formation of gas bubbles between electrodes was studied and there are several parameters which influence the process. The process parameters such as temperature, pressure, electrolyte concentration and input power are considered as the major parameters. Particularly for water electrolysis it was observed through experiments the coupled effect of parameters on the production rate of hydrogen. The aim of this paper is to develop a model correlation between volumetric production rate of hydrogen and above mentioned parameters. To understand the coupled effect of process parameters three dimensionless groups were derived through dimensional analysis.
\end{abstract}

Keywords: Electrolysis; Modeling; Coupled Effect

\section{INTRODUCTION}

Hydrogen is recognized and expected to be a useful alternative energy sources in the near future because of its absolute cleanliness, as it is not chemically bonded with carbon. Though several processes can generate hydrogen, still the acceptability of such processes was not reported because of techno-economic viability as well as sustainability. Among all these processes, water electrolysis for the production of hydrogen is getting importance because of its sustainability but it has number of drawbacks such as losses of input power, longevity of electrodes material and finding of optimum process parameters.

In this paper, therefore, a model on alkaline water electrolysis was established, incorporating the influences of temperature, applied pressure, electrolyte concentration and applied DC power in order to explain the effects of individual as well as coupled effect of pertinent parameters. Furthermore, water electrolysis experiments were conducted using potassium hydroxide $(\mathrm{KOH})$ aqueous solution and graphite electrodes. These experimental data were obtained for verifying the present model.

\subsection{Description of Water Electrolysis Model}

Considering the alkaline electrolysis for hydrogen production an electrolyzer cell starts producing gas bubbles instantly upon the application of DC input to the cell. In the initial phase of operation some gas may dissolve in media due to its solubility. Hydrogen and oxygen were produced from cathode and anode respectively. The gas bubbles are considered to rise up between electrodes. Increasing number of gas bubbles between electrodes subsequently reduces the local current density [1]. Upon reduction of operating pressure increases the partial pressure difference between gas phase and liquid phase, and increase in driving force for dissolution of gasses from electrolyte solution resulting increase in gas bubble rising velocity[2][3][4]. The volumetric production rate of hydrogen increases with increase in operating temperature, and this might be due to decrease in electrolyte viscosity which subsequently increases ion's mobility. Again, variation of electrolyte concentration significantly changes the electrolyzer's performance. Electrolysis starts upon the application of DC power to the electrolyzer, and input power played an important role on the production of hydrogen. Thus, the effects of pertinent parameters on volumetric hydrogen production can be further established by a model for alkaline electrolysis.

In this model on water electrolysis following situations were assumed.

[1]. Cell voltage is independent of the height of the electrodes.

[2]. Gas bubble rising velocity is considered to be uniform throughout the height of the electrodes.

[3]. No gas separator was introduced in the system.

[4]. Distance between two consecutive electrodes is constant.

[5]. Saturation of the system is already attained.

[6]. Reversible potential is independent of current density.

[7]. There is no interaction between $\mathrm{H}^{+}$and $\mathrm{H}_{2} \mathrm{O}$ molecules i.e., all $\mathrm{H}^{+}$ion is utilized for $\mathrm{H}_{2}$ production.

\subsection{Mathematical Model}

It is attempted to predict volumetric flow rate of hydrogen (Q) with respect to parameters such as temperature (T), pressure $(\mathrm{P})$, electrolyte concentration $(\mathrm{C})$ and input power to the electrolyzer (W). Volumetric flow rate of hydrogen (Q) is a function of T, P, C and $\mathrm{W}$ hence the general mathematical form appears as follows; 


$$
Q \propto f(T, P, C, W)
$$

$$
\begin{aligned}
& \text { Hence } \\
& \frac{Q}{Q_{o}}=k\left(\frac{T}{T_{o}}, \frac{P}{P_{o}}, \frac{C}{C_{o}}, \frac{W}{W_{o}}\right)
\end{aligned}
$$

Where, $T_{o}, P_{o}, C_{o}$ and $W_{o}$ represents the respective datum values.

Let $, M=\frac{Q}{Q_{o}}, x=\frac{T}{T_{o}}, y=\frac{P}{P_{o}}, z=\frac{C}{C_{o}}, t=\frac{W}{W_{o}}$

\section{Hence the equation reduces to}

$M=k\left(x^{a} y^{b} z^{c} t^{d}\right)$

$\ln M=\ln k+a \ln x+b \ln y+c \ln z+d \ln t$

Using linear least square technique we got

$\ln k=1.5473, a=3.3388, b=-0.6995$,

$c=0.0897, d=0.4709$

Hence the correlation appears as follows:

$\frac{Q}{\mathrm{Q}_{\mathrm{o}}}=4.698766\left(\frac{\mathrm{T}}{\mathrm{T}_{\mathrm{o}}}\right)^{3.3388}\left(\frac{P}{P_{o}}\right)^{-0.6995}\left(\frac{C}{C_{o}}\right)^{0.0897}\left(\frac{W}{W_{o}}\right)^{0.4709} \ldots$

This correlation is further validated using the experimental data sets and the attempt of model fitting is found to be fairly satisfactory $(85.3 \%)$. The experimental volumetric flow rate of hydrogen versus calculated volumetric flow rate of hydrogen was plotted as represented in fig. 1 .

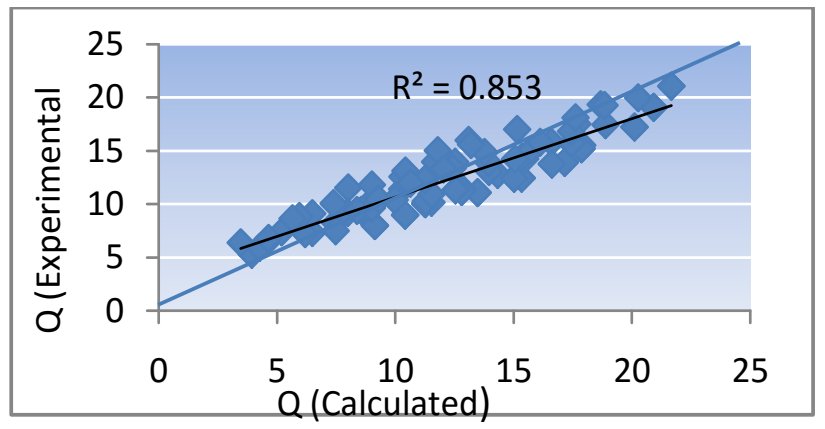

Fig-1: Plot of experimental volumetric hydrogen flow rate versus calculated volumetric hydrogen flow rate using the model correlation.

Since, the above correlation of hydrogen production rate by electrolysis to various parameters is srtictly an empirical one, an attempt has been made to develop a correlation based on dimensional analysis, leading to dimensionless groups which have physical significance of overall phenomena occuring due to various parameter's influences coupled together. Theoretically input DC power to electrolyzer was not utilized entirely and the losses of unutilized power appeared as rise in electrolyte temperature during electrolysis. Thus, in dimensional analysis of pertinent parameters, thermal resistance and distance between electrodes were introduced[5]. The process variables for electrolysis are $\mathrm{Q}, \mathrm{T}, \mathrm{P}, \mathrm{C}, \mathrm{L}, \mathrm{W}$ and $\mathrm{H}$ (volumetric hydrogen production rate, temperature, applied pressure, electrolyte concentration, applied power, distance between electrode and thermal resistance).T, L, C and $\mathrm{H}$ were considered as repeating variable, therefore, three dimensionless groups would appear to justify physical significance of the process.

Three dimensionless groups are as follows;

$$
\frac{Q H^{1 / 3} C^{1 / 3}}{T^{1 / 3} L^{4 / 3}}, \frac{H W}{T} \text { and } \frac{P H^{2 / 3} L^{4 / 3}}{T^{2 / 3} C^{1 / 3}}
$$

To establish the relationship between these dimensionless groups, following functional analysis was carried out.

$$
\begin{aligned}
& \left(\frac{Q H^{1 / 3} C^{1 / 3}}{T^{1 / 3} L^{4 / 3}}\right)=K\left(\frac{H W}{T}\right)^{a}\left(\frac{P H^{2 / 3} L^{4 / 3}}{T^{2 / 3} C^{1 / 3}}\right)^{b} \ldots \ldots \\
& \ln \left(\frac{Q H^{1 / 3} C^{1 / 3}}{T^{1 / 3} L^{4 / 3}}\right)=\ln K+a \ln \left(\frac{H W}{T}\right)+b \ln \left(\frac{P H^{2 / 3} L^{4 / 3}}{T^{2 / 3} C^{1 / 3}}\right) \ldots
\end{aligned}
$$

After calculation of coefficients and constant, the correlation appears as

$$
\left(\frac{Q H^{1 / 3} C^{1 / 3}}{T^{1 / 3} L^{4 / 3}}\right)=1.345\left(\frac{H W}{T}\right)^{1.141}\left(\frac{P H^{2 / 3} L^{4 / 3}}{T^{2 / 3} C^{1 / 3}}\right)^{-0.261}
$$

\subsection{Results and Discussion}

The correlation was attempted to validate with experimental results and the rader chart shows that with increase in temperature, volumetric production rate of hydrogen increases presented in fig. 2 .

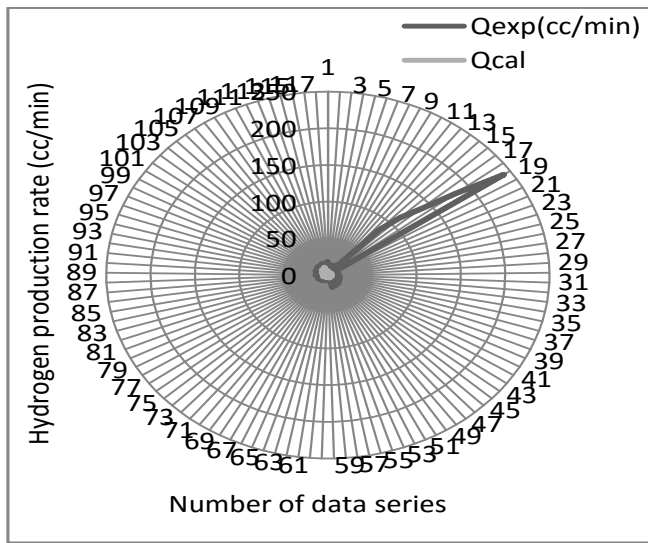

Fig-2: Rader chart of experimental volumetric hydrogen flow rate versus calculated volumetric hydrogen flow rate using the correlation of dimensionless groups. 
Again, it was observed that the decrease in applied pressure leads to increase in hydrogen production rate significantly and attained maximum at lowest operating pressure of 500 $\mathrm{mm} \mathrm{Hg}$ gauge. Moreover, it was noticed that hydrogen production rate increased with applied power to the electrolyzer. The effect of electrolyte concentration was not straight forward because of coupled effects. Increase in electrolyte concentration leads to increase in input power losses. Thus, the physical significance of three dimensional groups can be concluded as the ratio of forces. Dimensionless groups such as

$$
\frac{Q H^{1 / 3} C^{1 / 3}}{T^{1 / 3} L^{4 / 3}}, \frac{H W}{T} \text {, and } \frac{P H^{2 / 3} L^{4 / 3}}{T^{2 / 3} C^{1 / 3}}
$$

can expressed as the ratio of forces such as product flux/ heat loss flux, Power number and Ion mass flux/ applied power respectively. With increase in power number $\left(\frac{H W}{T}\right)$ within the range of 0.0033 to 0.036304 , the volumetric hydrogen production rate increases. Another dimensionless group shows the coupled effect of product flux and heat loss

flux $\left(\frac{Q H^{1 / 3} C^{1 / 3}}{T^{1 / 3} L^{4 / 3}}\right)$ which was checked to be valid within the ranges of 0.000271 to 0.090377 , within the present limits of investigations. The third dimensionless group $\left(\frac{P H^{2 / 3} L^{4 / 3}}{T^{2 / 3} C^{1 / 3}}\right)$ also reveals the coupled effect of ion mass flux and applied power on water electrolysis. With increase in applied power and ion mass flux within the range of 123.5297 to 1454.777 leads to increase in electrolyzer performance in terms of hydrogen production rate.

\section{CONCLUSION}

An alkaline aqueous electrolysis process model was established and succeeding in explaining the influences of temperature, pressure, electrolyte concentration, input power and thermal resistance on the hydrogen production rate. Three dimensionless groups were deduced to justify the couple effect of pertinent parameters and validated using experimental results.

\section{REFERENCES}

[1]. Nagai N., M. Takeuchi, M. nakao, "Influences of Bubbles between Electrodes onto Efficiency of Alkaline Water Electrolysis" Proceedings of PSFVIP4,June 3-5, 2003, Chamonix, France

[2]. Bongenaar-Schlenter B.E., L. J. J. Janssen, S. J. D. Vanstralen, E. Barendrecht, " The effect of the gas void distribution on the ohmic resistance during water electrolysis" Journal of applied electrochemistry, 15(1985)pp 537-548

[3]. Weijs M. P. M. G., L. J. J. Janssen, G. J. Visser, “ Ohmic resistance of solution in a vertical gas evolving cell" Journal of applied electrochemistry ,27(1997) pp371-378

[4]. Funk, J.E. and Thorpe, J.F., "Void Fraction and Current Density Distributions in a Water Electrolysis Cell”, J. Electrochem. Soc., Vol.116, (1969), pp.48-54.

[5]. Nagai, N., Takeuchi, M., Kimura, T. and Oka, T., "Existence of Optimum Space between Electrodes on Hydrogen Production by Water Electrolysis", Int. J. Hydrogen Energy, (2003), Vol.28, pp.35-41.

\section{BIOGRAPHY}

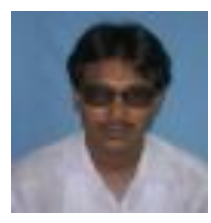

Biswajit Mandal is working as Assistant Professor in Chemical engg. Department at Haldia Institute of Technology for ten years.

Email Id: bmandal_1977@rediffmail.com

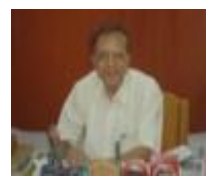

Prof. Amalesh Sirkar Is One Of The Renowned Researcher On Coal Liquefaction Process Is Working At HIT For 13 Years. He Owned Seven Patents On Different Fields Including Coal

Liquefaction Process.

Email Id: Sirkar_A@Hotmail.Com

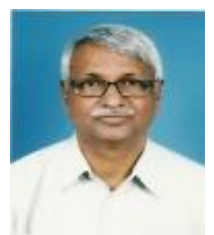

Prof. Parameswar De is Professor in the Department of Chemical Engineering at the University of Calcutta. He has engaged himself in teaching and research for over 30 years. His research areas include mass transfer \& hydrodynamics in multiphase flow, biochemical \& electrochemical engineering and reaction engineering. He has published papers in national and international journals.

Email Id: parameswar_de@rediffmail.com

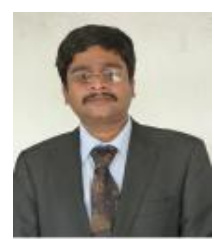

Dr. Somak Jyoti Sahu teaches at Haldia Institute of technology, Haldia. His current research focus on Process Modeling, Simulation and Control. He has more than ten research publication in International Journal and Conference

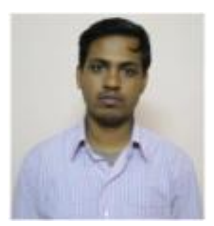

Dr. Avijit Ghosh, received his $\mathrm{PhD}$ degree from IIT Guwahati and M.Tech from Calcutta University. His research interest in the energy conversion device, graphene synthesis and its application, fuel cell. Presently, he is an assistant professor in Haldia Institute of Technology, Kolkata. His research paper is published in high impact factor peer review journals including fuel cells, international journal of hydrogen energy. 